\title{
Post-Partum Ilio-Psoas Abscess. A Rare Case of Puerperal Sepsis with Streptococcus Agalactiae
}

\author{
Huda Saleh*1, Rati Barman ${ }^{1}$ and Gamal Sayed ${ }^{1,2}$ \\ ${ }^{1}$ Department of Obstetrics \& Gynecology, Weil-Cornell Medical College in Qatar university, Qatar \\ ${ }^{2}$ Department of Obstetrics \& Gynecology, University of Dundee, UK
}

*Corresponding author: Huda Saleh, Department of Obstetrics \& Gynecology, Women's Wellness and Research Center/WeilCornell Medical College in Qatar university, Qatar.

To Cite This Article: Huda Saleh. Post-Partum Ilio-Psoas Abscess. A Rare Case of Puerperal Sepsis with Streptococcus Agalactiae. Am J Biomed Sci \& Res. 2019 - 4(3). AJBSR.MS.ID.000781. DOI: 10.34297/AJBSR.2019.04.000781

Received: : July 13, 2019 | Published: July 25, 2019

\section{Case Summary}

Mrs. A a 41 years old gravida 6 para 5 lady delivered vaginally, with spontaneous onset of labour at 39 weeks of gestation under epidural analgesia with intact perineum. She had a regular antenatal care at a large tertiary care center. Her antenatal risk factors were a high body mass index and mild anemia with a normal Glucose tolerance test and no other medical or surgical co morbidities. Day one post vaginal delivery she started complaining of left hip and back pain restricting her mobility. Her pain was of new onset and described as spasm like and starting from left hip and radiating to the whole of her left leg. An initial management plan was conservative in form of analgesia and although she had some relief from the pain aspect, yet she started complaining of lower abdominal pain, left back pain and left hip pain with radiation till the toes with inability to walk and presence of fever with chills.

Neurology consultation was done, and an urgent MRI performed which revealed edema and swelling in the left posterior Para spinous muscles at L4-S2 level. There was also edema and swelling noted in the left iliacus muscle and adjacent psoas muscle with extension to the pelvis. There were three small foci of collection noted in the inflamed left iliacus muscle; the largest one is measuring $13 \mathrm{~mm}$ in diameter. Minimal edema was noted in the left upper gluteus muscles. Minimal fluid collection noted in the left sacroiliac joint with marrow edema in the adjacent left iliac bone. Grade 1 anterolisthesis of L5 over S1 vertebra was noted. No significant degenerative changes in the lumbar spine was noted. No obvious epidural hematoma was noted.

InsummarytheMRI revealedinfectiveleftsacroiliitisand myositis involving the left posterior Para spinal, iliopsoas and upper gluteus muscles and three small foci of abscess seen in the inflamed left iliacus muscle Rheumatology consultation revealed Tinel test positive bilaterally, Negative straight leg test, No restriction of hip flexion, internal or external rotation. Tenderness over left paraspinal muscles and left buttock. It was felt that the low back pain is secondary to local infection to the paraspinal, iliopsoas, gluteus and iliacus muscle with small foci of abscess. Management included a multidisciplinary team approach including the obstetrician and Infectious disease physician, sepsis workup including blood culture, urine culture and genital culture. Parenteral ampicillin-sulbactam was started. The patient responded very well to parenteral antibiotics. She remained afebrile and her pain relief was adequate with paracetamol and diclofenac. She was discharged home in very good condition with follow up with mobile Doctor team to receive Injections of ceftriaxone once daily and for follow up MRI after 4 weeks (Table 1).

\begin{tabular}{|c|c|c|}
\hline WBC count & 6.1 & Normal \\
\hline Hemoglobin & $11 \mathrm{gm} / \mathrm{dl}$ & Normal \\
\hline Neutrophil count & $94 \%$ & Normal \\
\hline Blood urea & $4.7 \mathrm{mmol} / \mathrm{L}$ & Normal \\
\hline Serum creatinine & 56umol/L & Normal \\
\hline NT Pro BNP & 3,437 & $0-130$ \\
\hline Creatinine Kinase & $87 \mathrm{u} / \mathrm{L}$ & $29-168$ \\
\hline CRP & $460 \mathrm{mg} / \mathrm{L}$ & $0-5$ \\
\hline Serum lactate & $1.5 \mathrm{mmol} / \mathrm{L}$ & $0.5-2.2$ \\
\hline Procalcitonin & $3.22 \mathrm{ng} / \mathrm{ml}$ & Normal \\
\hline ANA CTD Int & Negative & \\
\hline Quantiferon TB & Indeterminate & \\
\hline Brucella Ab IgG and IgM & Negative & \\
\hline Blood culture aerobic & \multirow{2}{*}{\multicolumn{2}{|c|}{$\begin{array}{l}\text { Positive for Streptococcus Agalactiae, } \\
\text { sensitive to penicillin and ceftriaxone }\end{array}$}} \\
\hline Blood culture anaerobic & & \\
\hline Urine culture & \multicolumn{2}{|c|}{$\begin{array}{l}\text { Mixed growth including Streptococcus } \\
\text { Agalactaie }\end{array}$} \\
\hline
\end{tabular}




\section{Discussion}

Psoas (or iliopsoas) abscess is a collection of pus in the iliopsoas muscle compartment [1] and a rare cause of postpartum sepsis with a reported incidence of 0.4 per 100,000 population per year [2] Iliopsoas abscess may be classified as primary or secondary. Primary psoas abscess is thought to result from a lymphatic or haematological spread from an infection elsewhere in the body which maybe occult [3]. Risk factors include diabetes mellitus, intravenous drug use, AIDS, renal failure and immunosuppression [4] Crohn's disease is the commonest cause of secondary iliopsoas abscess [5]. Secondary psoas abscess originates from infection or inflammation in anatomically nearby structures with direct spread to psoas muscle. These structures include bone, and vertebrae, genitourinary and gastrointestinal structures, vessels and hip joint. As such, other conditions associated with secondary psoas abscess include diverticulitis, appendicitis, urinary tract infection, septic arthritis, intrauterine contraceptive devices, colorectal or genitourinary cancer [1]

The exact pathogenesis of psoas abscess following vaginal delivery in unknown. Perhaps it may be due to a hematoma formation following trauma in a vaginal delivery that later gets infected [6,7]. Psoas abscess has also been described as a rare complication of epidural anesthesia [8,9]. Streptococcus Agalactaie GBS vaginal colonization has been linked to the development of GBS psoas abscesses after delivery. This is a rare complication and is generally regarded as a secondary psoas abscess [10]. The presentation is insidious, at times non-specific and these vague symptoms can be attributed to other - commoner - causes in the postpartum period causing a diagnostic difficulty. Psoas abscess is usually unilateral, there is no significant difference between affection in either side left or right. The incidence is higher in males, with a male to female ratio of 3:1 Symptoms and signs of psoas abscess include pain, fever, limp, malaise, weight loss and lump in the groin. Pain can be localized to the back, flank, lower abdomen. Additionally, as the psoas muscle is innervated by L2, L3, and L4, pain can radiate to hip and posterior thigh [1]

The lower abdominal pain often is exaggerated on movements in which the Psoas muscle is extended or stretched. Psoas sign pain is brought about by extension of the hip. Patients prefer to acquire positions with less discomfort including hip flexion and lumbar lordosis. Limitation of the hip movement is common [11].

Due to nonspecific symptoms, slow subacute onset and overlap with other commoner pathologies, the median time between the onset of symptoms and actual diagnosis is 22 days. In a third of patients it may even take more than 42 days to reach the diagnosis [12]. The laboratory tests may show leukocytosis, elevated Creactive protein, elevated ESR and anemia [12]. Identification of the etiological organism requires culture of blood or aspirated pus. Numerous organisms can be responsible. The most common causative organism in primary iliopsoas abscess is Staphylococcus aureus responsible for over $88 \%$ of the cases [6] followed by Escherichia coli, Streptococcus Agalactaie, viridans streptococci, staph epidermidis, salmonella spp and Bacteroides species [12-14].
In the secondary abscess category, E. coli was the leading organism in this series, followed by S. aureus, Klebsiella pneumoniae, viridans streptococci and Candida albicans [14]. In our case the causative organism was Streptococcus Agalactaie. MRSA infections have also been described [13]. This all places emphasis on the need for a culture of aspirated pus to target the causative organism(s)

Acid-fast bacilli (AFB) smear and mycobacterial culture should also be performed when tuberculosis is suspected or when routine Gram staining is negative. Additionally, histopathology from the biopsy material should be sent as may suggest an alternate diagnosis as cancer [15]. The diagnosis of psoas abscess suspected on clinical grounds can be confirmed on imaging. Ultrasound and plain radiographs can be a useful primary investigation to exclude other causes that may explain the symptoms. Ultrasound is easy to perform and relatively inexpensive. Unfortunately, both are unhelpful to diagnose psoas abscess. Ultrasound has low sensitivity and specificity in cases of psoas abscess and is only diagnostic in about $60 \%$ of cases. Furthermore, ultrasound is largely operator dependent and It is difficult to visualize retroperitoneal structures when they are obscured by bowel and bone [16-19].

Magnetic resonance imaging (MRI) may allow improved definition of soft tissues and adjacent structures, especially visualization of the vertebral bodies $[1,16]$. CT scan however is regarded by many as the modality of choice or gold standard in diagnosis of psoas abscess when it is suspected clinically with reported sensitivity of $100 \%$ and specificity of $77 \%$. [1,12,20]. Management consists of surgical drainage and appropriate antibiotic therapy. Although its ideal to target the causative organism (s) after the results of culture and sensitivity, in practice we suggest empirical antibiotic therapy then modifying the antibiotic therapy according to the results of culture. A reasonable empirical antibiotics regimen should target Staphylococcus aureus being the main organism isolated from primary psoas abscess as well as Escherichia coli, streptococcus and Bacteroides species. Where MRSA is suspected or is of high local prevalence an appropriate antibiotic should be used.

Parenteral antibiotics should be administered in conjunction with Psoas abscess drainage $[1,18,20]$. Antibiotics are unlikely to be curative on their own although some success with antibiotic therapy alone is reported in abscesses less than $3 \mathrm{~cm}$ [21]. The optimal duration of antibiotics is uncertain but maybe required for 2-6 weeks following adequate drainage. The duration of therapy may be dictated by presence of concurrent osteomyelitis. Follow up imaging should be performed near the end of planned course of antibiotics to ensure satisfactory response to treatment $[16,18]$. The presence of Iliopsoas abscess is associated with significant morbidity and mortality, with mortality rate of 2.4 to 19 $\%$. In untreated cases it may be as high as $100 \%$ [1]. The risk is higher in cases with delayed or inadequate treatment, presence of bacteremia. There is a high chance of relapse, from $15-36 \%$. The high recurrence rate may be associated with inadequate drainage or inadequate antimicrobial therapy $[12,18]$. 


\section{Conclusion and Summary}

Psoas abscess is rare and if inadequately treated may lead to significant morbidity and mortality. Its rarity combined with insidious onset and the presentation that could be attributed to other causes can lead to delayed diagnosis. CT scan is a preferred imaging modality in diagnosis of psoas abscess. Main stay of treatment is both antibiotics and surgical drainage. Selected cases can be treated medically. Complete resolution can take few weeks.

\section{References}

1. I H Mallick, M H Thoufeeq, T P Rajendran (2004) lliopsoas abscesses. Postgrad Med J 80(946): 459-462.

2. Nabwera H, Gopalarnuragan AB, Snape J (2002) An unusual cause of hip pain. CME Journal Geriatric Medicine 4(3):125-126.

3. Lau SK, Woo PC, Yim TC, To AP, Yuen KY, et al. (2003) Molecular characterization of a strain of group a streptococcus isolated from a patient with a psoas abscess. J Clin Microbiol 41(10): 4888-4891.

4. Walsh TR, Reilly JR, Hanley E, Webster M, Peitzman A, et al. (1992) Changing etiology of iliopsoas abscess. Am J Surg 163: 413-416.

5. Agrawal SN, Dwivedi AJ, Khan M (2002) Primary psoas abscess. Dig Dis Sci 47(9): 2103-2105.

6. Garagiola DM, Tarver RD, Gibson L, Rogers RE, Wass JL, et al. (1989) Anatomic changes in the pelvis after uncomplicated vaginal delivery: a CT study on 14 women. AJR Am J Roentgenol 153(6):1239-1241.

7. Shah PN, Rane VA, Moolgaoker AS (1992) Retroperitoneal abscess complicating a normal delivery. Br J Obstet Gynaecol 99(2): 160-16.

8. Kraychete DC, Rocha AP, Castro PA (2007) Psoas muscle abscess after epidural analgesia: case report: Revista Brasileira De Anestesiologia [Rev Bras Anestesiol], ISSN: 1806-907X, Sociedade Brasileira de Anestesiologia, Brazil, 57(2): 195-198.

9. Flavin NE, Gomez M (2009) Fever, Pain, and a Limp: A Case of a Psoas and Spinal Epidural Abscess Caused by Methicillin-Resistant Staphylococcus aureus in a Diabetic Patient. J Natl Med Assoc 101(1): 84-86.
10. N Pandya, K Accardi, C Israelite (2006) Septic Arthritis of the Hip Following Group B Streptococcal Psoas Abscess in a Postpartum Patient Resulting in Total Hip Arthroplasty. The Internet Journal of Orthopedic Surgery 6(2): 1-6.

11. Stefanich RJ, Moskowitz A (1987) Hip flexion deformity secondary to acute pyogenic psoas abscess. Orthop Rev 16(2): 67-77.

12. Navarro Lopez V, Ramos JM, Meseguer V, Pérez Arellano JL, Serrano R, et al. (2009) Microbiology and outcome of iliopsoas abscess in 124 patients. Medicine (Baltimore) 88(2): 120-130.

13. Sokolov KM, Kreye E, Miller LG, Choi C, Tang AW, et al. (2007) Postpartum iliopsoas pyomyositis due to community-acquired methicillin-resistant Staphylococcus aureus. Obstet Gynecol 110(2 Pt 2): 535-538.

14. Lin MF, Lau YJ, Hu BS, Shi ZY, Lin YH, et al. (1999) Pyogenic psoas abscess: analysis of 27 cases. J Microbiol Immunol Infect. 32(4): 261-268.

15. Kishi Y, Kajiwara S, Seta S, Kawauchi N, Suzuki T, et al. (2002) Retroperitoneal schwannoma misdiagnosed as a psoas abscess: report of a case. Surg Today 32(9): 849-852.

16. Lee YT, Lee CM, Su SC, Liu CP, Wang TE (1999) Psoas abscess: a 10 year review. J Microbiol Immunol Infect 32(1): 40-46.

17. Ricci MA, Rose FB, Meyer KK (1986) Pyogenic psoas abscess: worldwide variations in etiology. World J Surg 10(5): 834-843.

18. Huang JJ, Ruaan MK, Lan RR, Wang MC (2000) Acute pyogenic iliopsoas abscess in Taiwan: clinical features, diagnosis, treatments and outcome. J Infect 40: 248-255.

19. Gordin F, Stamler C, Mills J (1983) Pyogenic psoas abscesses: noninvasive diagnostic techniques and review of the literature. Rev Infect Dis 5(6): 1003-1011.

20. Amy Kwan et al (2009) Iliopsoas abscess: an unusual cause of postpartum sepsis Obstet Med 2(1): 30-31.

21. Yacoub WN, Sohn HJ, Chan S, Petrosyan M, Vermaire HM, et al. (2008) Psoas abscess rarely requires surgical intervention. Am J Surg 96(2): 223-227. 